\title{
Nano-thin 2D Soft Materials - Design Principles and Prospects
}

\author{
Mykhailo Vybornyia ${ }^{\mathrm{a}}$, Hao Yư , and Robert Häner ${ }^{\star \star}$
}

\begin{abstract}
Established less than a decade ago, the fascinating field of two-dimensional (2D) soft materials is advancing continuously towards widespread recognition. After demonstrating the feasibility of obtaining nano-thin supramolecular sheets and morphologically related tubular objects, considerable efforts are being undertaken to explore the functional potential of soft nanosheets. Self-assembly is a major tool for the controlled formation of nanometre-sized 2D objects. In this account, we share our current understanding of the structural requirements to direct the self-assembly of water-soluble, negatively charged oligomers in 2D. The discussion covers some promising areas of utilization such as the reporting of weak mechanical perturbations, the assembly of lightharvesting antennae, the transfer of excitation energy and the polymerization of pre-organized assemblies. The systems presented in this work illustrate the potential of 2D supramolecular materials as complementary systems to their covalent counterparts.
\end{abstract}

Keywords: 2D polymer $\cdot$ Nanosheet $\cdot$ Nanotube $\cdot$ Self-assembly $\cdot$ Supramolecular polymer

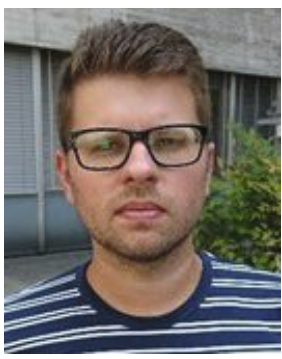

Mykhailo (Misha) Vybornyi got his BSc from the Kyiv National University (Ukraine) and MSc from the University of Angers (France) in nanotechnology and organic materials. In 2011, he joined the Häner group at the University of Bern (Switzerland) to work on the development of synthetic oligomers for 2D supramolecular polymerization. As an SNF postdoctoral fellow, he conducted research with Prof. E.W. (Bert) Meijer at the Eindhoven University of Technology (the Netherlands). In 2018, Mykhailo moved to the industrial sector (Enamine, Kyiv, Ukraine). Since 2019, he has been a member of Prof. Andrew Griffiths' group at the ESPCI in Paris.

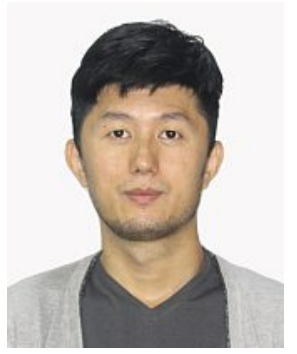

Hao Yu obtained his PhD in polymer chemistry under the supervision of Prof. A. Dieter Schlüter in 2015 at the ETH Zürich. Afterwards, he joined the group of Prof. Robert Häner at the University of Bern and worked as a postdoc for 3 years. In 2018, he returned to his homeland China and started his professional career as an associate professor at Wuhan University. Now he is working in the area of novel polymeric materials, including their precisely controlled synthesis and characterization on the molecular level.

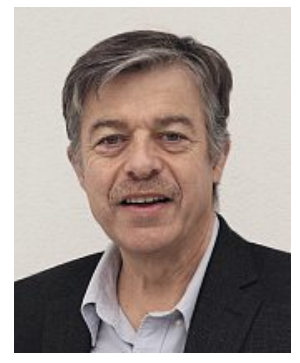

Robert Häner received his $\mathrm{PhD}$ in chemistry in 1987 from the ETH Zurich, Switzerland. After three years of postdoctoral work at UC Berkeley and California Institute of Technology, he worked in Swiss pharmaceutical research in 1990, where he held several positions in Sandoz, Ciba and Novartis. Since 2000 he has been full professor of organic chemistry at the University of Bern, Switzerland. His research interests focus on the understanding of the fundamental aspects of supramolecular organization of DNA-based nanomaterials and the application of modified nucleic acids in the fields of diagnostics and novel materials.

\section{Introduction}

Two-dimensional materials have received an enormous attention over the past decade. ${ }^{[1-3]}$ The interest in such morphologically defined matter stems from the theoretical considerations supported by vast experimental data acquired from the most eminent member of 2D materials - graphene. ${ }^{[4]}$ Preparation of organic nanometre-thin sheets remains among the most challenging aspects in material and polymer sciences because it will provide access to technological applications. Exciting opportunities for this type of materials emerge in areas such as molecular separation, ultralight and strong protective coats, electronics or medicine.

Comprehensive synthetic methodologies toward organic 2D polymers have been developed in several research groups. ${ }^{[5,6]}$ Nowadays, monomers for 2D polymerization have become commercially available, which is a positive sign for establishing multidisciplinary collaborations between research and industrial groups. ${ }^{[7]}$ This and other ground-breaking contributions were achieved by Schlüter and co-workers who inspired other research groups to join the field. ${ }^{[8]}$ The Schlüter group solved a number of highly challenging problems on their way to bringing $2 \mathrm{D}$ polymers from scientifically curious to technically valuable materi- 
als. In one of the most successful strategies, 2D polymerization was realized in a crystalline phase, enabling covalent connection of monomers within a plane. ${ }^{[9]}$ Reducing the thickness of sheets to a few nanometres, i.e. going to a single layer, is realized by an exfoliation procedure. However, the necessity of possessing a crystalline material is - and probably will remain - a limiting factor in diversifying molecular scaffolds suitable for 2D polymers. The example further highlights the importance of pre-ordering to achieve the proper arrangement of monomers within 2D confined materials. At this time, the possibility to prepare free-standing organic nanosheets, in which the building elements are connected via noncovalent bonds, was barely described in the literature. Facile access to these systems should lead to the benefits of using them as stimuli-responsive and adaptable materials, as well as other characteristics shared by supramolecular polymers. ${ }^{[10]}$

Overall, 2D soft materials have been largely overshadowed by their fibrillar counterparts, similar to the situation established between $1 \mathrm{D}$ and 2D polymers a decade ago. While working on DNA-inspired materials, our group contributed to the conceptual understanding of some basic principles enabling the preparation of 2D soft materials, i.e. 2D supramolecular polymers. Here, we describe the major steps undertaken in our laboratories during recent years.

\section{Free-standing 2D Supramolecular Materials}

Our original efforts were directed at defining the necessary structural requirements to escape the one-dimensional supramolecular polymerization by extending the self-assembly to the $2 \mathrm{D}$ space. Theoretically, this change can be implemented by using a monomer which forms more than two directional non-covalent bonds, similar to the approach for covalent $2 \mathrm{D}$ polymerization. Oligomeric units represent ideal substrates for this purpose due to their multivalency. After formation of 2D assemblies, however, their further integration into multilayer aggregates must be restricted. By working in aqueous environment, the latter restriction can be efficiently realized by exploiting the strong repulsive forces between charged groups, e.g. phosphates located at the surfaces of the formed sheets. To this end, our group has engaged in the preparation of various sequence-specific and monodisperse oligophosphodiesters. Such oligomers are readily accessible by automated solid phase synthesis using phosphoramidite chemistry. ${ }^{[11]}$ Originally developed for the synthesis of nucleic acids, this chemistry perfectly suits our needs for preparation of oligomers like the ones shown in Fig. 1.
The first oligomer shown to form 2D systems was trimer $\mathbf{1}$ composed of a 1,6-bis-substituted pyrene, in which the units are linked by phosphodiester bonds. ${ }^{[12]}$ The self-assembly of $\mathbf{1}$ occurs via strong stacking and hydrophobic interactions in aqueous solution and leads to $2 \mathrm{~nm}$ thin sheets, as proved by AFM and TEM analysis (Fig. 2A). Insight into the molecular packing of pyrene units within 2D sheets was obtained from UV-vis and fluorescence spectroscopy. J- and H-bands were observed for two electronic pyrene transitions $(\mathrm{S} 0 \rightarrow \mathrm{S} 1$ and $\mathrm{S} 0 \rightarrow \mathrm{S} 2$ ), which can be correlated with the relative orientation of the calculated transition dipole moments of pyrenes within the aggregates. In addition, the intensity of pyrene excimer emission decreases upon self-assembly into 2D supramolecular polymers, suggesting considerable steric constraints within stacked pyrenes. Overall, these data support a model of ladder-type folded trimers of $\mathbf{1}$ that self-assemble into a nanosheet. The use of a controlled assembly protocol allowed the preparation of free-floating sheets with lateral dimensions exceeding a few tens of micrometres while retaining a constant thickness of $2 \mathrm{~nm}$. Such large free-standing sheets were obtained upon slow cooling of $\mathbf{1}$ from high temperatures to ambient conditions. ${ }^{[13]}$ Apart from the cooling rate, also proper buffering conditions and the controlling of the ionic strength were among the major variables ensuring stacking in 2D. The morphological differences observed in the assembly of isomeric pyrenes incorporated in similar oligomers hint at the molecular folding pattern of the oligomeric construction units as a major factor for directing the self-assembly in 2D. Thus, an oligomer 2 consisting of 2,7-bisalkynyl substituted pyrenes follows a similar assembly pathway leading to 2D sheets as the major product. ${ }^{[14]}$ At the same time, oligomer 2 can also grow into nanotubes (see below). In contrast, oligomer $\mathbf{3}$, which is based on a 1,8-bisalkynyl substituted pyrene and a 3,6-bisalkynyl phenanthrene self-assembles in 1D supramolecular fibres.[15]

The research on monodisperse oligomers was further extended to other systems. Thus, the number of pyrenes within an oligomer chain was increased from three to seven. The heptamer 4 behaved similarly, yielding 2D supramolecular polymers. An important step toward enhancing the functional potential of $2 \mathrm{D}$ supramolecular polymers resides in the preparation of chimeric oligomers. To this end, a series of oligomers containing a nucleobase conjugated to a heptamer was successfully tested for $2 \mathrm{D}$ supramolecular polymerization. ${ }^{[16]}$ This observation opens the door to the preparation of water-soluble planar platforms with a high density of small molecules on its surface. However, increasing the
Fig. 1. Structures of molecular building blocks and illustrative representation of the corresponding oligomers. Oligomers were prepared via solid-phase synthesis.

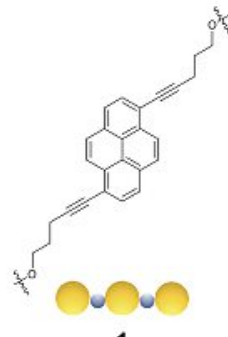

1
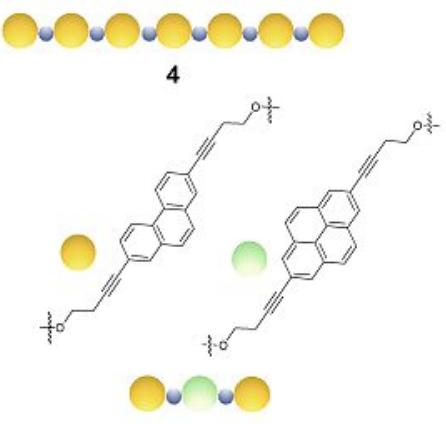

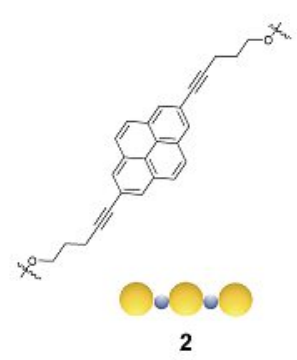

2
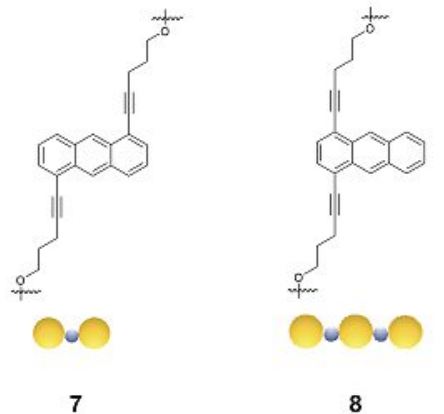

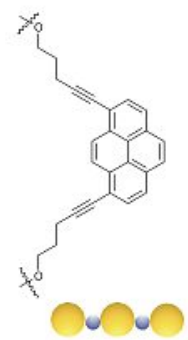

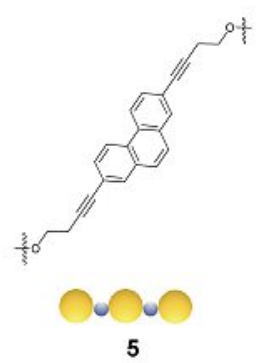

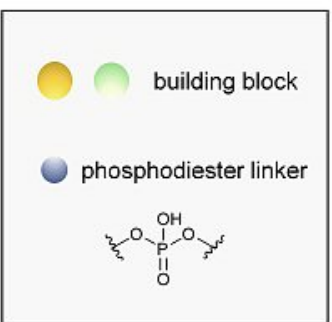


A

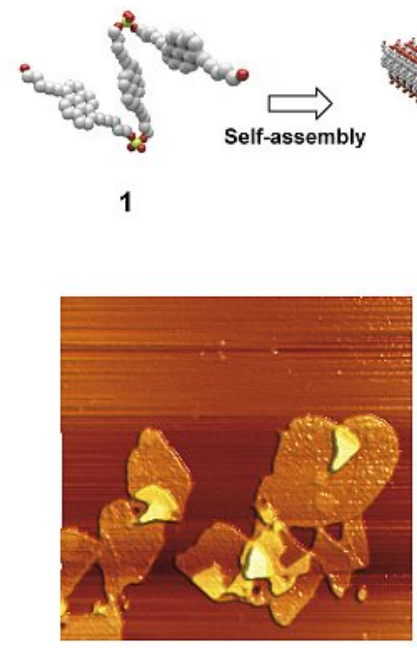

B

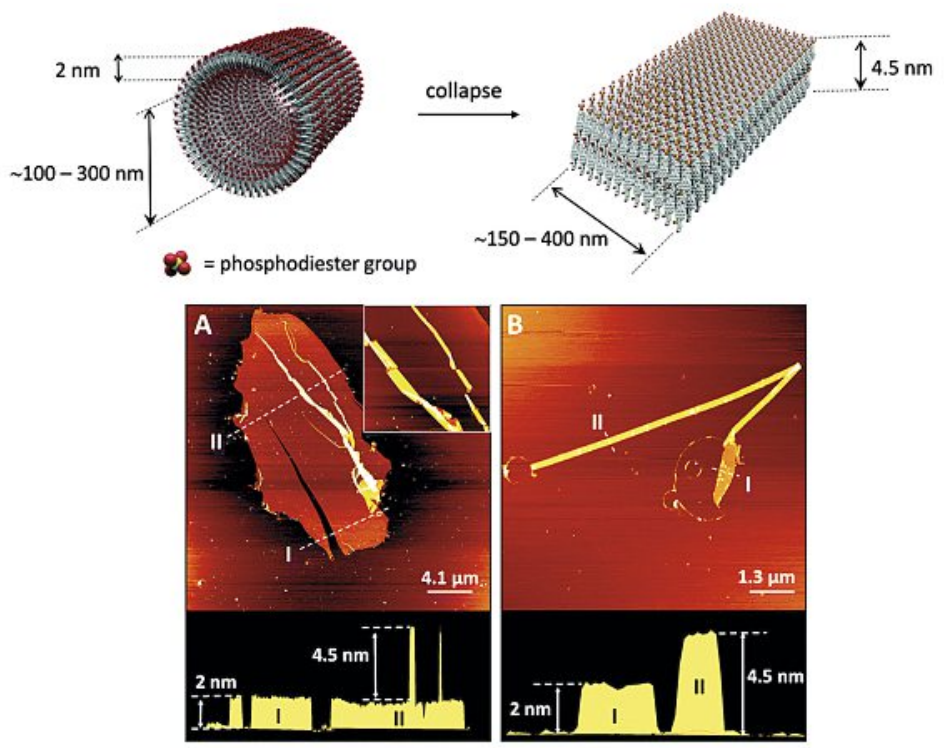

Fig. 2. (A) Illustration of the self-assembly of 1 into 2D supramolecular polymers and a representative AFM image nanosheets adsorbed on mica (adapted from ref. [13]). (B) AFM visualization of self-assembled 2 (adapted from ref. [14]). AFM images reveal formation of sheets, as well as nanotubes. Reprinted with permission from the corresponding references (copyrights Wiley, RSC and ACS).

number of nucleobases along the oligomer chain led to the formation of 1D supramolecular polymers, indicating a structural limitation to the approach of constructing $2 \mathrm{D}$ functional platforms.

Chirality in soluble 2D supramolecular polymers remains an almost entirely unexplored area. Some recent results highlight the possibilities to get optically active sheets either by integrating chiral units within the assembled polymers or via applying subtle mechanical perturbations (internal flows). In the former case, the activity was manifested by emerging CD signals in the area of pyrene absorption. ${ }^{[16]}$ In the latter case, vortexing of the solution led to the emergence of a strong chiroptical readout.[17] This method can be used for the detection or visualization of internal flows.

\section{2D Motifs in Tubular Systems}

Often, the assignment of dimensionality in materials is rather arbitrary. For example, tubular objects can be regarded as quasione-dimensional at the microscopic level. However, many properties at the nanoscale will be more related to $2 \mathrm{D}$ materials, especially when the walls of a tube consist of organic molecules. In one of the reports, we observed the divergence of self-assembly pathways for 2 in supramolecular sheets and tubes. ${ }^{[14]}$ For the surface-collapsed tubes, AFM analysis revealed the thickness of $4.5 \mathrm{~nm}$, which cor- relates with two layers of stacked pyrenes (see Fig. 2B). Owing to the cooperative mechanism of supramolecular polymerization, these morphologically related shapes can co-exist due to the dynamic and reversible interactions during the growth of assemblies in 2D. This example highlights our limited understanding of the mechanistic aspects of $2 \mathrm{D}$ supramolecular polymerization. Further research in the area is needed to advance the applications of $2 \mathrm{D}$ materials, including light harvesting and energy transfer. To this end, supramolecular copolymerization of oligomers 5 and $\mathbf{6}$ yields exclusively tubular objects and allowed the creation of a light-harvesting system (Fig. 3). ${ }^{[18]}$ In stark contrast to covalent polymers, successful integration of two oligomers in tubes becomes possible due to the dynamic nature of supramolecular polymers and close structural similarity of the oligomers. Once $\mathbf{5}$ and $\mathbf{6}$ are included in tubes, 2,7-bis-substituted phenanthrenes can absorb light and subsequently transfer energy over long distances to pyrenes doped in the nanotubes. The high efficiency of these light-harvesting nanotubes is based on properly ordered chromophore stacks. Again, dynamic interactions between constituent molecular units facilitate correction of defects, if such are present, and can offer superior properties over covalent materials.

Inspired by the elegantly manifested usefulness of anthracenes for construction of $2 \mathrm{D}$ polymers, some of us explored the
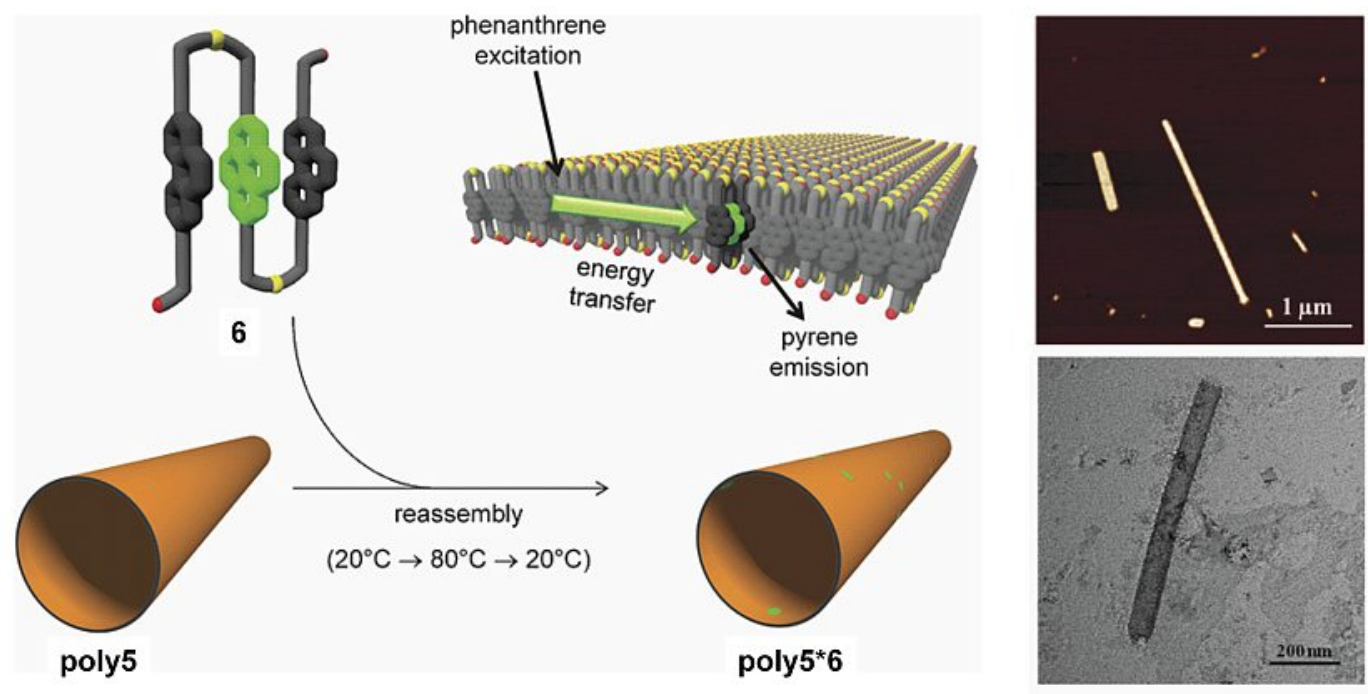

Fig. 3. Light-harvesting nanotubes prepared by co-polymerization of $\mathbf{5}$ and $\mathbf{6}$ with illustrative microscopic images (adapted from ref. [18]). Reprinted with permission; copyright Wiley. 
potential of oligomers containing this chromophore to undergo supramolecular polymerization. ${ }^{[19]}$ Thus, dimer 7 consisting of 1,5-bis-substituted anthracenes self-assembles into nanotubes. The morphology was confirmed by a set of spectroscopic and microscopic experiments. Furthermore, photoinduced crosslinking of anthracenes provided important insights into their internal organization within the nanotubes. Most probably, the assembly occurs via end-to-end stacking of folded dimers. This conclusion is supported by the post-crosslinking AFM imaging, which confirmed the absence of long polymers in ethanol, in which solvent oligomer $\mathbf{7}$ is soluble. The case underlines that substitution pattern of crosslinking units, determining folding and assembly modes, is vital for obtaining $2 \mathrm{D}$ covalent polymers. In the next chapter, we will highlight successful cases of obtaining covalent 2D materials starting from supramolecular polymers.

\section{From 2D Supramolecular Polymers to 2D Covalent Materials}

To arrive at covalent 2D materials, we turned our attention to DNA scaffolds which have been widely employed to create nano-objects of various morphologies. Pioneered by Seeman et $a l .$, chemists have greatly succeeded in the pursuit of creating 2D nano-architectures from DNA single strands. ${ }^{[20]}$ In our work, we designed a DNA-based 2D network that can be internally crosslinked by covalent bonds. ${ }^{[21]}$ The Y-shaped monomer contains a benzene core, to which three identical, self-complementary DNA single strands are attached at 1-, 3- and 5- positions, respectively. For the later linkage formation, an 1,4-bis-substituted anthracene unit is incorporated at the middle of each oligonucleotide branch. When the monomers self-assemble into planar 2D networks with hexagonal repeat units, the duplex formation will bring anthracene units together as face-to-face stacked pairs, which can be dimerized by UV irradiation (Fig. 4). The formation of 2D supramolecular polymers (2D-SP) was achieved by careful annealing of monomers in an aqueous solution. AFM images identified large, homogeneous sheets with randomly distributed cracks and holes. The measured thickness of $\sim 1 \mathrm{~nm}$ is in good agreement with the height of a DNA duplex adsorbed on mica which indicates the formation of a monolayer sheet. The solution of nanosheets was exposed to UV light at $366 \mathrm{~nm}$. The anthracene absorption decreased over time and reached a final conversion of $83 \%$ into dimers after 12 hours. The resulting 2D polymer (2DP) resembles its supramolecular precursor in shape and thickness, though small holes are observed. Furthermore, an interesting solvent-responsivity has been observed when transferring 2DP from water to acetonitrile (Fig. 5).

In different work, entirely non-nucleosidic, anthracene-based oligomers such as $\mathbf{8}$ can be used for the polymerization via pre-organized, supramolecular polymers. ${ }^{[22]}$ In aqueous solution, the ol- igomer self-assembles into tubular structures. Internal ordering of anthracenes favours photo-induced dimer formation and consequently leads to covalent tubes (Fig. 6).

Apart from organic materials, the negatively charged 2D supramolecular polymers, e.g. 1, can be used as templates for the growth of inorganic materials. ${ }^{[23]}$ We demonstrated that using water-soluble 2D scaffolds opens the door to nanothin silicates. First, a positively charged reagent, methyl[3-(trimethoxysilyl)propyl] ammoniumchloride, is added to create nucleation sites on the nanosheet surface. Second, introduction of triethoxysilane, which is easily hydrolysed in aqueous buffer at $\mathrm{pH}=7$, leads to the slow covering of nanosheets with a silica shell (Fig. 7). The thickness of the shell can be controlled by the amount of added silane.

\section{Conclusions and Outlook}

Two-dimensional soft matter is a fascinating topic of research, holding exciting promises for future applications. Several important milestones in this field, such as demonstration of oligomer self-assembly in soft 2D materials and development of reliable preparation methods, have already been achieved. Currently, the major problems lying ahead include the optimization of the preparation protocols, extending the scope of suitable small molecules or oligomers, and exploiting multicomponent assemblies as a way to functional diversification. The latter type of $2 \mathrm{D}$ materials is still elusive and the field of supramolecular polymerization would greatly benefit from developments in this direction. With further advances in the field, such systems can be used as integral components of mimicking multifunctional biomaterials. Thus, it was shown that inorganic nanoscale coatings, e.g. silicates, can be grown on the surfaces of 2D soft materials and used for further derivatization. Finally, soluble 2D supramolecular polymers can be used as precursors for covalent polymerization, overcoming some limitation of modern synthesis of $2 \mathrm{D}$ polymers.

\section{Acknowledgements}

Financial support by the Swiss National Foundation (grant 200020_169030) is gratefully acknowledged.

Received: February 25, 2019

[1] P. Miro, M. Audiffred, T. Heine, Chem. Soc. Rev. 2014, 43, 6537.

[2] J. W. Colson, W. R. Dichtel, Nat. Chem. 2013, 5, 453.

[3] C. Tan, X. Cao, X. J. Wu, Q. He, J. Yang, X. Zhang, J. Chen, W. Zhao, S. Han, G. H. Nam, M. Sindoro, H. Zhang, Chem. Rev. 2017, 117, 6225.

[4] A. Geim, K. Novoselov, Nat. Mater. 2007, 6, 183.

[5] S. L. Cai, W. G. Zhang, R. N. Zuckermann, Z. T. Li, X. Zhao, Y. Liu, Adv Mater. 2015, 27, 5762 .

[6] X. Feng, A. D. Schlüter, Angew. Chem. Int. Ed. 2018, 57, 13748.

[7] http://www.polychem.mat.ethz.ch/research/Technical_scale_synthesis.html.

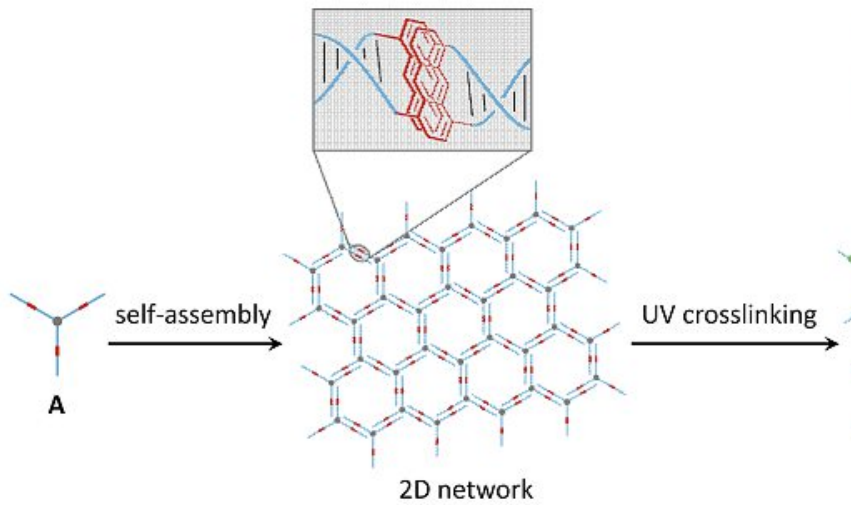

$2 \mathrm{D}$ network
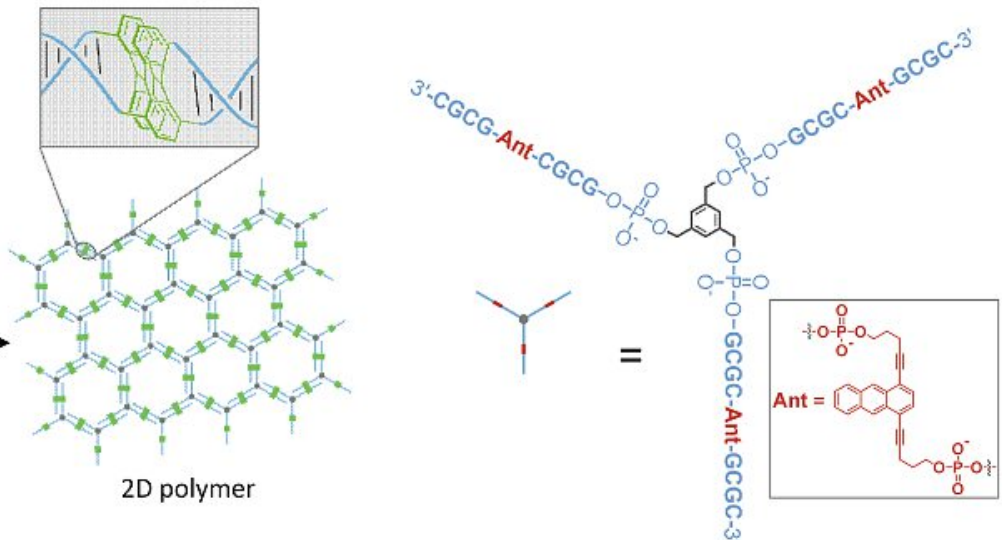

Fig. 4. Cartoon illustration of the formation of 2D supramolecular networks and 2D polymers by assembly and crosslinking of anthracene modified tri-valent oligonucleotide A (adapted from ref. [21]). Reprinted with permission; copyright Wiley. 
in acetonitrile

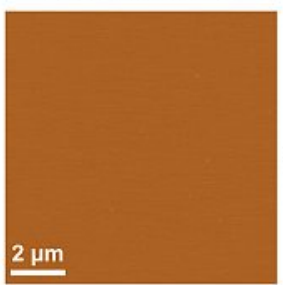

no aggregate

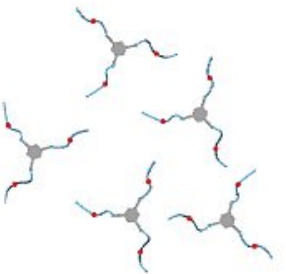

A in water

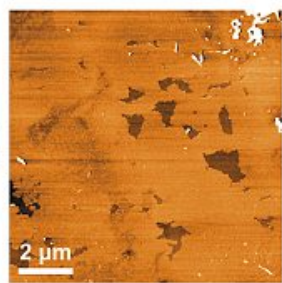

2D-SP

thin sheet

height $\sim 1 \mathrm{~nm}$

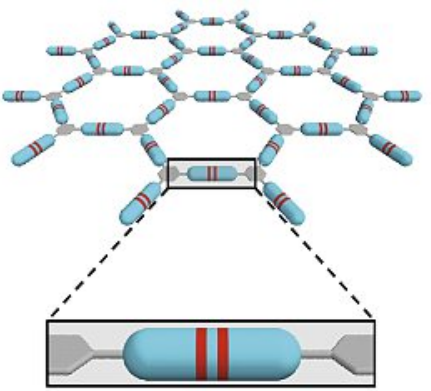

in water

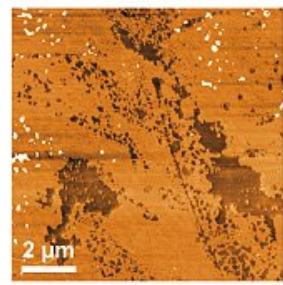

2DP

thin sheet

height $\sim 1 \mathrm{~nm}$

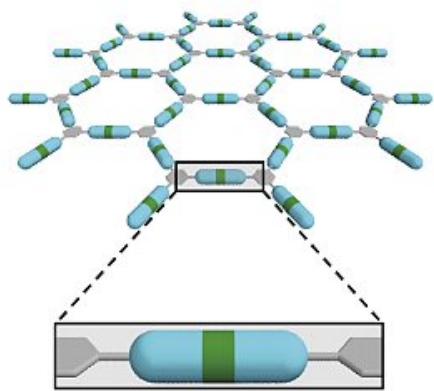

in acetonitrile

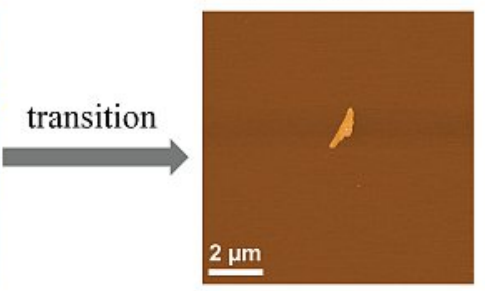

2DP

thick pad

height $4-5 \mathrm{~nm}$

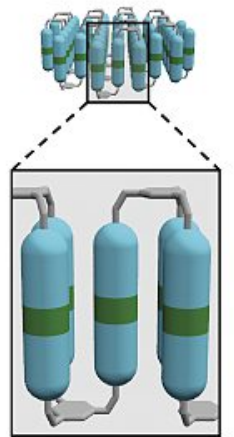

Fig. 5. AFM images of monomer A, supramolecular polymer (2D-SP) and covalent polymer after anthracene photo-crosslinking (2DP) in different solvents (top), and their proposed models (bottom, adapted from ref. [21]). Reprinted with permission; copyright Wiley.
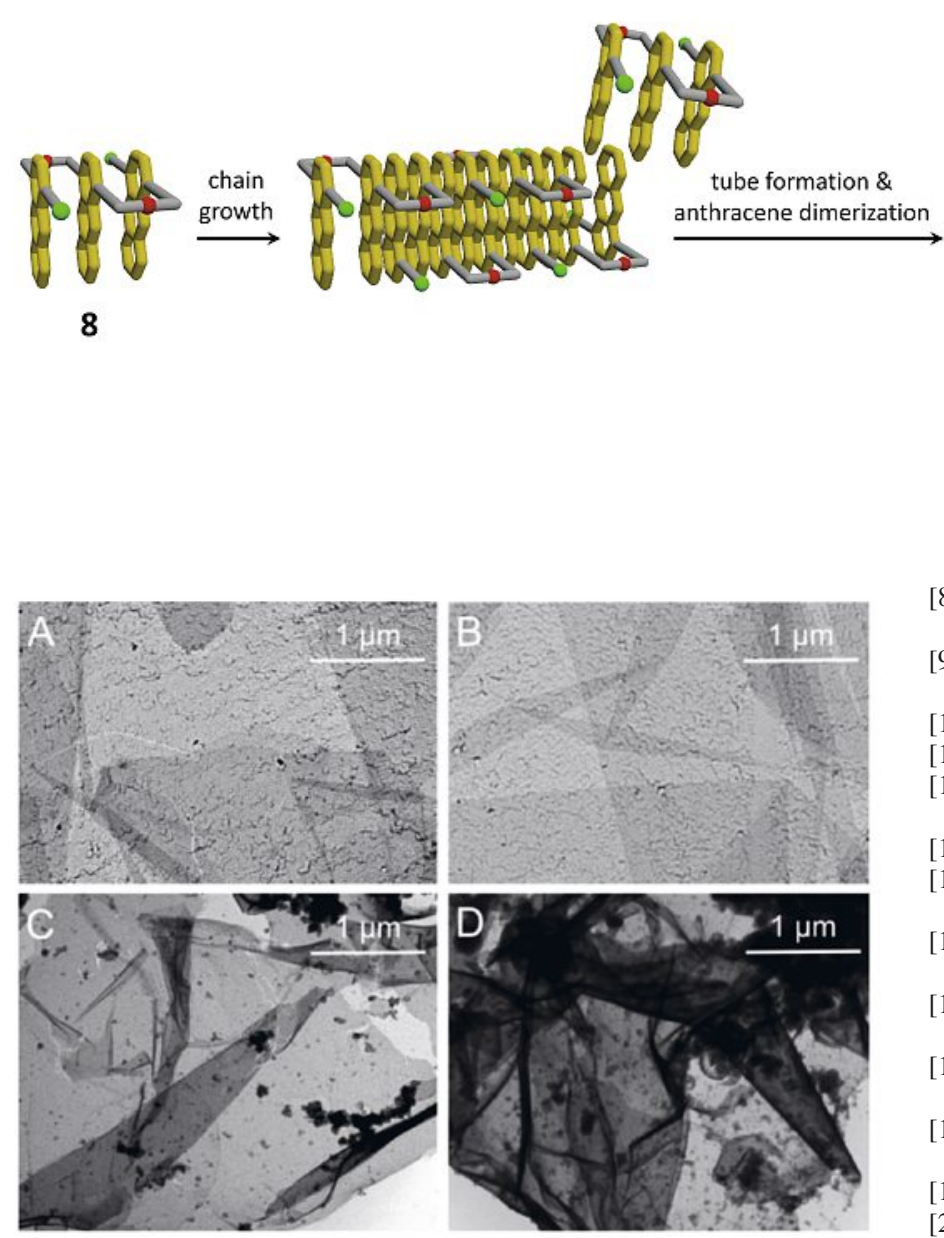

Fig. 7. TEM images of nanosheets from 1 before (A, B) and after (C, D) growing a silica shell around the SP sheets (adapted from ref. [23]). Reprinted with permission; copyright Wiley.

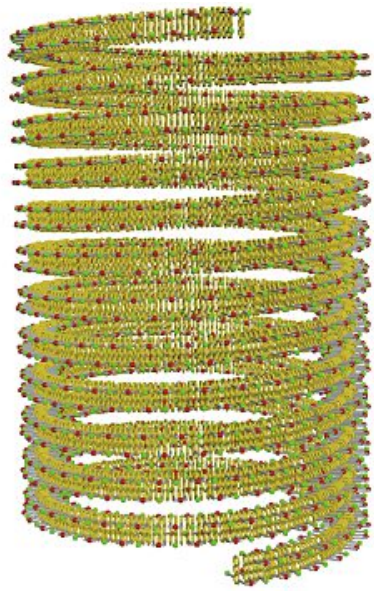

Fig. 6. Oligomer 8 self-assembles into a linear supramolecular polymer, followed by folding into a nanotube, which is further converted into a covalent polymer by anthracene photo-crosslinking (adapted from ref. [22]). Reprinted with permission; copyright RSC.

[8] P. Kissel, R. Erni, W. Schweizer, M. D. Rossell, B. T. King, T. Bauer, S. Goetzinger, A. D. Schlüter, J. Sakamoto, Nat. Chem. 2012, 4, 287.

[9] M. J. Kory, M. Woerle, T. Weber, P. Payamyar, S. W. van de Poll, J. Dshemuchadse, N. Trapp, A. D. Schlüter, Nat. Chem. 2014, 6, 779.

[10] T. Aida, E. Meijer, S. I. Stupp, Science 2012, 335, 813.

[11] M. H. Caruthers, Science 1985, 230, 281.

[12] M. Vybornyi, A. V. Rudnev, S. M. Langenegger, T. Wandlowski, G. Calzaferri, R. Häner, Angew. Chem. Int. Ed. 2013, 52, 11488.

[13] M. Vybornyi, A. Rudnev, R. Häner, Chem. Mater. 2015, 27, 1426.

[14] M. Vybornyi, Y. B.-C. Hechevarria, M. Glauser, A. V. Rudnev, R. Häner, Chem. Commun. 2015, 51, 16191.

[15] C. B. Winiger, S. Li, G. R. Kumar, S. M. Langenegger, R. Häner, Angew. Chem. Int. Ed. 2014, 53, 13609.

[16] Y. Vyborna, S. Altunbas, M. Vybornyi, R. Häner, Chem. Commun. 2017, 53, 12128.

[17] N. Micali, M. Vybornyi, P. Mineo, O. Khorev, R. Häner, V. Villari, Chem. Eur. J. 2015, 21, 9505.

[18] C. D. Bösch, S. M. Langenegger, R. Häner, Angew. Chem. Int. Ed. 2016, 55, 9961.

[19] H. Yu, M. Sabetti, R. Häner, Chem. Asian J. 2018, 13, 968.

[20] E. Winfree, F. Liu, L. A. Wenzler, N. C. Seeman, Nature 1998, 394, 539.

[21] H. Yu, D. T. L. Alexander, U. Aschauer, R. Häner, Angew. Chem. Int. Ed. 2017, 56, 5040.

[22] H. Yu, R. Häner, Chem. Commun. 2016, 52, 14396.

[23] M. Vybornyi, Y. Vyborna, R. Häner, Chemistry Open 2017, 6, 488. 